Vol. 24, No. 02, pp.112-122/Diciembre 2011

\title{
Asentamientos poblacionales periféricos más seguros. Instrumental para el diseño y evaluación de la calidad ambiental
}

\author{
F. del Pozo* y G. Gómez \\ Facultad de Arquitectura. Universidad de Oriente. Santiago de Cuba. Cuba \\ e-mail: greycu@yahoo.es
}

(Recibido/received: 08-Agosto-2011; aceptado/accepted: 28-Noviembre-2011)

\begin{abstract}
RESUMEN
La vulnerabilidad de un asentamiento ante cualquier situación y ámbito territorial, es una preocupación, debido entre otras cuestiones a su fuerte influencia en las políticas de desarrollo de gestión frente a desastres o eventos adversos externos que afectan el normal desarrollo de las personas; de ahí que diagnosticar la calidad ambiental de una urbanización en áreas espaciales específicas, como es el caso de las urbanizaciones periféricas, posibilita analizar aquellos factores que influyen en la vulnerabilidad de estas y formular recomendaciones para implementar políticas ambientales que mitiguen los riesgos. Partiendo de que la vulnerabilidad es una noción dinámica que examina los factores de riesgos, se presenta un instrumental para la evaluación ambiental de los Asentamientos Poblacionales Periféricos (APP), en las condiciones particulares de la ciudad Santiago de Cuba, donde se ha considerado esta componente dentro de sus indicadores evaluativos. Se expone el instrumental de referencia y su sistema categorial: variables, subvariables; parámetros evaluativos y escala de valores, lo que constituye la esencia del instrumental teórico diseñado; de igual forma se presentan los histogramas y matrices que conforman el instrumental práctico de evaluación. El mismo servirá para evaluar asentamientos en funcionamiento y para el diseño de nuevos, pudiendo ser generalizado a otros territorios.
\end{abstract}

Palabras claves: Asentamientos poblacionales periféricos, calidad ambiental, vulnerabilidad.

\begin{abstract}
The vulnerability of settlement before any situation and territorial scope, is a concern, due inter alia to their strong influence on the development policies of management to disaster or adverse external events affecting the normal development of the people; Summary to diagnose the environmental quality of urbanization in a discrete space, as it is the case of the peripheral housing estates, allows analyze those factors that influence the vulnerability of these and make recommendations for implementing environmental policies that mitigate the risks. On the basis of that vulnerability is a dynamic concept that examines the risk factors, continues an instrumental for environmental assessment of settlements population peripherals (APP), in particular the conditions of the city Santiago de Cuba, where it has considered this component in its evaluative indicators. You expose instrumentation reference and his categorical system: variable, subvariables, evaluative parameters and scale of values, which is the essence of the instrumental designed theorist; likewise are histograms and arrays that comprise the instrumental assessment workshop. It will serve to assess settlements in operation and for the design of new ones, and can be generalized to other territories.
\end{abstract}

Key words: Peripheral population settlements, environmental quality, vulnerability

\footnotetext{
${ }^{*}$ Autor para la correspondencia
} 


\section{F. del Pozo y Gómez G.}

\section{INTRODUCCIÓN}

El creciente aumento de los desastres en las áreas urbanas como consecuencia de causas naturales y antrópicas, ha derivado en daños al ambiente, es decir a todos los componentes del medio social y natural. Ante los diferentes eventos que pueden ocasionarse, los impactos sociales y naturales son menores si se adoptan las medidas desde el planeamiento o el reordenamiento de las urbanizaciones. Puede asegurarse además, que la gestión del riesgo sólo puede alcanzarse mediante una nueva visión cultural que incluya a todos los actores sociales que actúen frente a la problemática.

En relación al proceso de urbanización actualmente existe un sostenido proceso de urbanización en los países de América Latina, las ciudades crecen a un ritmo mayor que la capacidad para este crecimiento, no obstante, no debe preocupar el tamaño que alcanzarán las ciudades, sino la manera como están creciendo. La población que habita las ciudades crece y ocupa de manera espontánea el suelo urbano, y de ese modo crece también la vulnerabilidad física y social.

Es propósito de este trabajo, aportar un instrumental de evaluación de la calidad ambiental que contiene indicadores integrales para Asentamientos Poblacionales Periféricos (APP) existentes, cuyo resultado posibilita la gestión de las intervenciones en el medio construido, pertinentes para su seguridad y sustentabilidad. Es oportuno señalar la posibilidad de considerar los indicadores del instrumental para el planeamiento de nuevos APP.

Un Asentamiento Poblacional Periférico, es un asentamiento humano espontáneo o planificado que se encuentra ubicado próximo a los límites urbanos de la ciudad, cuyas características políticas, económicas o de densidad poblacional, no lo sitúan como asentamiento urbano, pero debido a su localización y crecimiento deberán ser estudiados, reorganizados o reubicados para su posterior fusión con la ciudad.

\section{MARCO TEÓRICO}

En general, en América Latina se adopta la definición de los criterios cuantitativos y cualitativos, o la combinación de ambos. Lo urbano se entiende como una entidad específica, un lugar con sus propias características y atributos, un medio físico con su propio ambiente. Pero además, lo urbano es un carácter, y como lo afirmó Castells, (1988), es hasta una ilusión; es decir, el sitio donde se pueden satisfacer las necesidades básicas de los que la habitan.

Ramírez, (2003) afirma "que las ciudades se han convertido en el centro dinámico de la vida nacional, donde la población se concentra, crece y se desarrolla. Donde se obtienen los servicios necesarios para su mantenimiento y reproducción y donde encuentran diversas posibilidades de empleo y producción. También se han convertido en serios problemas, que deben enfrentarse para lograr una vida digna y agradable para sus habitantes."

Es usual, que se considere urbano lo que los diferentes países definen como tal, según razones muy diversas: dimensión de la población, función administrativa, naturaleza de las actividades económicas, tradición histórica, infraestructuras, entre los aspectos relevantes. La escala a la que se refieren cada una de las concepciones de lo urbano no está planteada, entonces, puede ser desde un barrio, una unidad habitacional, una sección urbana, o hasta una mega ciudad con millones de habitantes concentrados.

Es preciso entonces comprender y aprovechar las particularidades que asume la ciudad en cada país y en cada territorio, entender mejor las relaciones, su forma de articulación, características de la población y su modo de vida, concretándose en esta investigación que la comprensión de lo urbano indica una pluralidad de requisitos y de criterios, cuyo emplazamiento debe de cumplir exigencias de densidad y ocupación del suelo, los rasgos particulares inherentes a grupos humanos en un territorio dado, con un modo de vida y aspectos ambientales particulares.

Como parte de las relaciones sociales, económicas y de identidad, se ha estado experimentando una nueva sensibilización en la sociedad hacia lo que es propio y constituye la raíz de la cultura del lugar, donde el modo de vivir, asume un rol principal, a ese particular refiere Echarri, (2001), "Rescatar el patrimonio cultural ayuda a conocer, donde surgen los modos de entender la realidad y el fundamento de las costumbres, un quicio sobre el cual deberá guiar todo lo que se pretende hacer en el presente y en el futuro". En consideración a lo antes expresado, las soluciones que se adopten para el hábitat, deben dar respuesta a costumbres, gustos y preferencias de los pobladores, lo que favorecerá a preservar la cultura propia de cada urbanización, así como las restantes características del ambiente, incluyendo las vulnerabilidades. 


\section{F. del Pozo y Gómez G.}

El concepto de calidad ambiental, tiene una definición ideal compleja, por lo tanto, al determinar la aplicación práctica del concepto, es algo que requiere de mucho esfuerzo para evitar que su aplicación sea restringida, de acuerdo a determinadas situaciones específicas, ya que en algunos casos la calidad ambiental es determinada en dependencia de ciertas actividades antrópicas o componentes del ambiente específicos, o de forma aislada.

En este trabajo se consideran además de los componentes del medio construido, aquellos de la naturaleza y la sociedad que se consideran esenciales, siendo este un aspecto distintivo y que tendrán su materialización en el conjunto de variables esenciales que se deberán determinar.

Se ha abordado la expresión de desarrollo sustentable con el significado de mejorar la calidad de vida humana, sin alterar la capacidad de carga de los ecosistemas, refiriéndose al desarrollo general de estos, lo que incluye lo concerniente a lo cultural, habiéndose pronunciado al respecto diversas organizaciones como: La Unión Internacional para la Conservación de la Naturaleza (UICN), el Programa de las Naciones Unidas para el Medio Ambiente (PNUMA), el Fondo Mundial para la Naturaleza (WWF), referido en el documento "Cuidar la tierra para el futuro de la vida",(1993); también se han manifestado al respecto, la Cumbre del Medio Ambiente Johannesburgo, (2002) e investigadores como Zhang, (2002) y Bancrofft, (2002).

Sin embargo, los sistemas habitacionales, en muy pocos países presentan un grado razonable de integralidad, la que implica sustentabilidad y apropiabilidad, es decir, desarrollo real, parejo y universal, debiendo ser una aspiración en el funcionamiento de los Asentamientos Poblacionales Periféricos, siendo en Cuba una premisa, por el tipo de sociedad.

Actualmente las ciudades y otros sistemas de asentamientos humanos son consumidores de recursos provenientes del medio natural y depositan en éste los desechos que en ellos se producen, lo que conduce al agotamiento de recursos y a la contaminación ambiental que caracteriza al mundo actual. Para transitar hacia la sustentabilidad, es necesario transformar esta situación buscando reutilizar lo que se desecha y producir lo que se necesita. Expresando resumidamente lo anterior, se define como el desarrollo que satisface las necesidades del presente, sin poner en peligro la capacidad de las generaciones futuras para atender sus propias necesidades. Se precisa que el desarrollo sustentable debe orientarse a la capacidad de conservar y ampliar las opciones disponibles en el asentamiento para confrontar un mundo natural $y$ social en permanente transformación.

La problemática existente en los APP en América Latina y Cuba, constituyen una importante referencia para conocer la situación que presenta la calidad ambiental en los mismos. El crecimiento urbano ha adquirido un carácter desordenado, incontrolado, casi cancerígeno, en tan solo 65 años; señala la Comisión Mundial del Medio Ambiente y del Desarrollo (CMMAD, 1988), "la población urbana de los países en desarrollo se ha decuplicado". Si en 1900 sólo un $10 \%$ de la población mundial vivía en ciudades, 2007 fue el primer año de la historia con más personas viviendo en áreas urbanas, según señaló el informe de Naciones Unidas UN- hábitat: el estado de las ciudades 2006-2007, el estallido demográfico urbano es un reto sin precedentes para la sociedad del siglo XXI, previendo que el $75 \%$ de la población vivirá en áreas urbanas en el año 2050. Por tanto, se tendrá el reto de ciudades que utilizan alrededor de un $75 \%$ de los recursos mundiales y desalojan cantidades semejantes de desechos Girardet, (2001).

Por las razones antes expuestas, se destaca que América Latina en general, está en un proceso de urbanización y entre las múltiples consecuencias de esta, no se puede olvidar que las concentraciones urbanas se caracterizan por originar problemas ambientales específicos Dávila, (2008).

Todo asentamiento humano provocará una demanda ambiental a los recursos ambientales (agua, suelo, materias primas, aire o energía) que les sirven de sustento, así como por la contaminación inherente a los vertidos de gran parte de estos recursos en forma de residuos (aguas residuales urbanas, basuras, y otros) o la propia expansión física originando la ocupación irreversible de suelo; aspectos que se suman a los problemas ambientales de los asentamientos poblacionales; según Gómez, (2005); en América Latina, uno de los problemas más trascendentales relacionados con los asentamientos humanos, es la interacción vivienda-ambiente.

El proceso de traslado del campesino a la ciudad, la migraciones; es la acción provocada por la desesperanza en la que vive en el campo y que no tiene recursos para acceder a una vivienda digna, puesto que lo embarga la pobreza, decidiendo instalarse espontáneamente, en una área de alta vulnerabilidad y sumar a la demanda existente e insatisfecha de servicios y equipamientos 


\section{F. del Pozo y Gómez G.}

básicos, agudizando más los problemas ya existentes y quizás en condiciones más precarias que en el campo.

La pobreza y el deterioro ambiental son las más relevantes características de las zonas periféricas en los países de Latinoamérica. Algunos de los fundamentales problemas que presenta la calidad ambiental en estos asentamientos vienen dados por:

- Escasa protección ambiental del territorio.

- Deterioro de ecosistemas y del paisaje.

- Contaminación de aguas y suelos.

- Malas condiciones de salubridad e higiene en las viviendas y los asentamientos.

- Carencia de infraestructuras técnicas de acueducto y tratamiento de residuales.

- Estado de deterioro de infraestructuras viales.

- Carencia de servicios sociales básicos.

- Déficit de viviendas, con alta promoción de viviendas en regular y mal estado.

Esta situación ha conllevado a que los países busquen alternativas que pongan freno a los grandes deterioros a los que se enfrentan, no siendo siempre posible, por no ser una pretensión de las direcciones de los Estados en los países latinoamericanos.

En Cuba, si bien la problemática no es la misma que en Latinoamérica, existen problemas que afectan la calidad ambiental en el sector de periferia, a pesar de las medidas adoptadas por el Estado desde el triunfo de la Revolución, en relación a garantizar viviendas dignas, servicios básicos y programas sociales que han contribuido al mejoramiento de la calidad de vida.

El ordenamiento y planificación del territorio juegan un papel decisivo, debido a las particularidades $\mathrm{y}$ necesidades de desarrollo del país, existiendo políticas para el desarrollo urbano, las cuales se deben tener presentes a la hora de planificar un área o territorio; hoy en día las áreas que se planifican han ocasionado en la mayoría de las veces un aumento desmedido de la problemática habitacional, debido al no completamiento de los asentamientos, y la proliferación de las viviendas ilegales. Según estudios realizados por la Dirección Provincial de Planificación Física (DPPF) de Santiago de Cuba, en la actualidad no cuentan con acciones urbanísticas necesarias para su organización, existiendo un predominio elevado de precariedad de las redes técnicas y los servicios en los pocos casos que lo tienen.

Existen Asentamientos Poblacionales Periféricos diseñados y otros surgidos espontáneamente, tanto en unos como en otros, se pueden enumerar algunos de los principales problemas del medio construido, que influyen en la calidad ambiental, cuestión que sería necesario investigar:

- Signos de deterioro de los ecosistemas.

- Paisajes naturales y antrópicos, con baja calidad escénica.

- Pérdida del patrimonio cultural.

- Carencia de un plan de ordenamiento.

- El deficitario sistema de instalaciones socioculturales.

- Carencia o mal estado de infraestructura de acueducto y tratamientos de residuales.

- Mal o regular estado técnico de la red vial, lo que dificulta la conectividad de los asentamientos con la ciudad y el territorio.

- Fondo habitacional poco confortable e inadecuadas soluciones constructivas.

Esta situación reafirma la necesidad de diagnosticar de forma sencilla, la problemática que afecta la calidad ambiental, en este caso específico en los APP en la ciudad de Santiago de Cuba, para accionar con inmediatez en esta problemática del medio construido con enfoque sistémico y de sustentabilidad.

Es objetivo del instrumental que se presenta para aspirar a un equilibrio ambiental de los APP, la consideración de indicadores que evalúen la problemática ambiental y garanticen la gestión frente a desastres o eventos adversos externos que afectan el normal desarrollo de la población.

\section{METODOLOGÍA}

Instrumental de evaluación de la problemática que afecta al hábitat urbano periférico

Los teóricos del urbanismo, actualmente hacen énfasis en la necesidad de aportar criterios para el proceso de medición de la calidad ambiental, al analizar la falta de herramientas que permitan a planificadores del desarrollo urbanístico evaluar ambientalmente un determinado territorio. La necesidad de un instrumental que ayude a evaluar ambientalmente el medio construido en Asentamientos Poblacionales Periféricos (APP), resultará de vital interés para el territorio y país, a continuación se presenta el instrumental de referencia, exponiéndose la forma de selección de los indicadores, el sistema categorial asumido, los componentes del instrumental teórico y práctico, así como su 


\section{F. del Pozo y Gómez G.}

procedimiento de aplicación, el que puede ser adecuado a otros territorios.

Criterios generales y proceso metódico para la determinación de los indicadores más significativos

El sistema de indicadores que se detalla a continuación es una guía metodológica para evaluar ambientalmente los APP en la ciudad de Santiago de Cuba. Para determinar los mismos, sus parámetros evaluativos y las escalas de valores a aplicar, se adoptan las siguientes consideraciones:

- Balancear la complejidad de los conceptos de calidad ambiental y sustentabilidad formulados por prestigiosos investigadores, centros y organizaciones de investigación de esta temática, para determinar indicadores simples, que resuman la información esencial para la evaluación.

- Retomar los datos que resultan válidos de las metodologías de evaluación revisadas.

- Aplicar las normas existentes en el país y los datos aportados por el territorio con relación al tema.

- Considerar los aportes de los especialistas en torno a las prioridades locales de los asentamientos.

- Adoptar las opiniones aportadas por los actores institucionales y comunitarios, ajustando alcances y contenidos de los indicadores, fusionándose o excluyéndose algunos componentes.

- La observación de la realidad y la valoración de estudios anteriores de calidad de vida realizados.

En la incorporación de los indicadores a considerar, se presentaron algunos desafíos a los cuales hace referencia Gómez, (2005) y esta a su vez a Bancrofft, (2002); Zhang, (2002) y Liepach, (2003). Entre éstos desafíos se destacan:

- Seleccionar indicadores simples y de fácil aplicación.

- Limitar los indicadores a considerar, con la finalidad de garantizar la simplicidad del instrumental.

Estos desafíos posibilitan la simplicidad y efectividad del instrumento.

\section{Sistema categorial del instrumental}

Los componentes que conforman el sistema para la evaluación, utilizándose los siguientes:

- Factores, variables y subvariables.

- Parámetros evaluativos.

- Escala de valores.
En el instrumental que se presenta, se adopta una estructura particular, a pesar de retomarse algunos componentes correspondientes a los utilizados por los venezolanos Contreras y Cordero, (1994) y luego utilizado por Gómez, (2005); Zúñiga, (2008) y Hernández, (2008), por poseer una organización de fácil comprensión y un sistema categorial que facilita el proceso evaluativo. Las categorías asumidas, son precisadas y adecuadas para este trabajo.

\section{Estructura del instrumental}

La elaboración de un instrumental para la evaluación ambiental del medio construido en APP; con criterios de sustentabilidad, es el fundamento de este trabajo, el mismo quedó conformado por un instrumental teórico y otro práctico. Para la elaboración del instrumental de referencia, se consultó a especialistas con categoría científica y/o docente en el campo de los asentamientos humanos, con amplias posibilidades de emitir criterios importantes, de igual forma se utilizó la bibliografía más actual en el campo de instrumentos de evaluación existentes.

Los indicadores para la evaluación se determinaron, a partir de la información aportada por los instrumentales analizados, la obtenida en los estudios a asentamientos poblacionales; así como de los criterios aportados por normativas, especialistas, actores institucionales vinculados a escenarios urbanos, así como de la propia comunidad que habita en estos asentamientos y en la que repercuten de forma aceptable o no, aquellos aspectos que inciden en su calidad ambiental.

\section{Determinación de las variables}

La fundamentación para la determinación de las variables, es a partir de los componentes esenciales del asentamiento, donde se fusionan todos aquellos componentes que se interrelacionan, posibilitando una evaluación sencilla y organizada, a los que se han referido, Aguiló, (1993); Bancrofft, (2002); Enviroment Building New, (1999), así como Guías y reglas de intervención en el planeamiento.

A continuación se describen los principales componentes del sistema de indicadores generales asumidos para la evaluación:

1. Funcionalidad del asentamiento: En esta variable se agrupan los aspectos que garantizan los requisitos organizacionales indispensables de tipo funcional, ecológico, de sanidad y estética, a escala del asentamiento. Son incluidos los componentes que 


\section{F. del Pozo y Gómez G.}

determinan una adecuada organización funcional del mismo, su posibilidad de crecimiento, orientación, reciclaje de desechos, aprovechamiento de energías renovables, estabilidad del asentamiento ante fenómenos naturales y antrópicos, mobiliario urbano, la relación con la ciudad y su sistema de asentamientos, por ser donde se encuentran los restantes servicios y fuentes de empleo.

2. Asentamiento y su entorno natural: En esta variable se considera la sanidad ambiental, por corresponder a esta una serie de componentes de elevada repercusión en el asentamiento, los relacionados con la contaminación. De igual forma se consideran la infestación por vectores y la adecuación al contexto con énfasis en la topografía, dándose además un elevado interés al área verde del sitio.

3. Percepción social del asentamiento: Es contemplada la calidad visual de los componentes naturales y antrópicos del asentamiento, las organizaciones sociales y su papel en el asentamiento, la producción de alimentos en la localidad, las tradicionales sociales y los valores patrimoniales.

4. Sistema de servicios sociales: Por la importancia y complejidad de los servicios sociales en el asentamiento, están considerados aquellos servicios imprescindibles para el funcionamiento del mismo; comercio y gastronomía, salud, educación, culturales, deportivos y recreativos.

5. Sistema de infraestructuras técnicas: Es considerada la existencia y estado de las redes viales, hidráulicas, eléctricas, de comunicaciones, así como la evacuación y tratamiento de los residuales y las aguas pluviales.

6. Características de las viviendas: Evalúa uno de los componentes esenciales del asentamiento: la vivienda. Según Aroztegui, citado por González, (2000), "la calidad de una vivienda está referida a su aptitud de habitar con la satisfacción de sus ocupantes", incorpora además, que "la calidad de la vivienda debe mantenerse durante toda su vida, introduciendo el mantenimiento y la conservación que se produce por deterioro y nivel de requerimiento del usuario". Notario, (1987), añade, "que la vivienda debe ofrecer seguridad, confort, eficiencia y durabilidad". Se consideran los requisitos de funcionabilidad, confort ambiental, sanidad, soluciones técnicas y estado constructivo, facilidad de mantenimiento, atendiendo a la posibilidad de adquisición de materiales, valores arquitectónicos y su adecuación al sitio, así como su comportamiento sismo resistente.

\section{RESULTADOS Y DISCUSIÓN}

El instrumental de evaluación quedó constituido por los anteriores componentes y estructurado en dos partes que se complementan para el análisis: la teórica y la práctica. Instrumental teórico de evaluación

El instrumental teórico quedó conformado por un sistema de variables y subvariables, que deberán ser considerados al efectuar cualquier valoración de la calidad ambiental en un APP. El mismo fue elaborado a partir del marco metodológico, normativo, y de los criterios aportados por especialistas y actores. (Ver tabla No.1).

\section{Instrumental práctico de evaluación}

Al instrumental teórico conformado por el sistema de indicadores: variables y subvariables, propuestos para la evaluación de los componentes de la calidad ambiental del medio construido en los APP de Santiago de Cuba, se le incorporó un procedimiento avalado por procesos matemáticos, que posibilitan una aplicación objetiva del mismo y que representa el instrumental práctico.

Para obtener el valor de la incidencia de la calidad ambiental en los asentamientos, será necesario obtener el valor de todas las variables.

Se ha propuesto para sintetizar los valores cuantitativos de las variables, la elaboración de histogramas, considerando el comportamiento de las subvariables que las conforman. Para sintetizar los valores cuantitativos de la evaluación de estas y por tanto de la calidad ambiental en el asentamiento se elabora la matriz síntesis, los que constituyen el instrumental práctico.

Se realizan histogramas, por ser gráficas estadísticas que permiten expresar valores agrupados en intervalos. Estas gráficas poseen reconocido uso en diversas temáticas; en la materia objeto de estudio, tiene como referencia, el modelo de evaluación de calidad de vida Gómez, (2005), siendo probado en varios estudios de casos de calidad de vida realizados y dirigidos por la citada investigadora, cuestión por la que se asume como instrumento evaluativo en esta investigación.

En cuanto a la matriz síntesis, es una forma de representación gráfica, que con el empleo de colores, ayuda a la evaluación final del comportamiento de los 


\section{F. del Pozo y Gómez G.}

componentes del medio construido. En esta investigación se ha diseñado de una forma precisa, respondiendo al sistema de indicadores adoptados.

Tabla No.1. Variables y subvariables

\begin{tabular}{|c|c|}
\hline VARIABLES & SUBVARIABLES \\
\hline $\begin{array}{l}\text { 1. FUNCIONALIDAD DEL } \\
\text { ASENTAMIENTO }\end{array}$ & 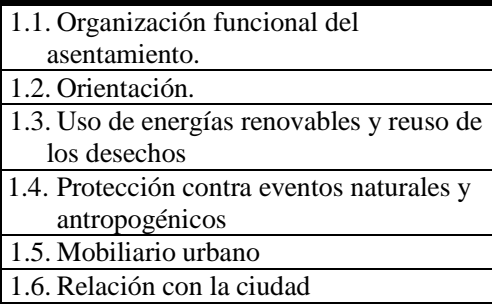 \\
\hline $\begin{array}{l}\text { 2. ASENTAMIENTO Y SU } \\
\text { ENTORNO NATURAL }\end{array}$ & \begin{tabular}{|l|} 
2.1. Estado del aire \\
2.2. Estado del agua \\
2.3. Estado del suelo \\
2.4. Áreas verdes \\
2.5.Topografía \\
2.6. Infestación por vectores \\
\end{tabular} \\
\hline $\begin{array}{l}\text { 3. PERCEPCIÓN SOCIAL } \\
\text { DEL ASENTAMIENTO }\end{array}$ & $\begin{array}{l}\text { 3.1. Valores paisajísticos } \\
\text { 3.2. Organizaciones sociales } \\
\begin{array}{l}\text { 3.3. Producción de alimentos en la } \\
\text { localidad }\end{array} \\
\text { 3.4. Tradiciones sociales } \\
\text { 3.5. Valores patrimoniales }\end{array}$ \\
\hline $\begin{array}{l}\text { 4. SISTEMA DE SERVICIOS } \\
\text { SOCIALES }\end{array}$ & $\begin{array}{l}\text { 4.1.Salud } \\
\text { 4.2. Educación } \\
\text { 4.3.Comercio y gastronomía } \\
\text { 4.4. Cultura, deporte y recreación }\end{array}$ \\
\hline $\begin{array}{l}\text { 5. SISTEMA } \\
\text { INFRAESTRUTURAS } \\
\text { TECNICAS }\end{array}$ & \begin{tabular}{|l} 
5.1.Redes hidráulicas \\
5.2.Redes viales \\
5.3.Redes de evacuación y tratamientos de \\
residuales, sistema de drenaje pluvial \\
5.4. Redes eléctricas \\
5.5.Sistemas de comunicaciones \\
\end{tabular} \\
\hline $\begin{array}{l}\text { 6. CARACTERISTICAS DE } \\
\text { LAS VIVIENDAS }\end{array}$ & $\begin{array}{l}\text { 6.1 Superficie construida útil en } \mathrm{m}^{2} \text {. } \\
\text { Progresividad } \\
\text { 6.2. Confort ambiental } \\
\text { 6.3.Condición higiénico-sanitarias } \\
\text { 6.4. Soluciones técnico-constructivas. } \\
\begin{array}{l}\text { 6.5.Relación con los puntos de adquisición } \\
\text { de materiales para la construcción }\end{array} \\
\begin{array}{l}\text { 6.6. Valores arquitectónicos e integración } \\
\text { al sitio }\end{array} \\
\text { 6.7. Comportamiento sismo resistente. }\end{array}$ \\
\hline
\end{tabular}

Fuente: Tabla elaborada por Del Pozo, Junio 2010

\section{Desarrollo de los histogramas}

Los histogramas son los gráficos que representan el comportamiento de cada variable. Para su confección es necesario tener un conocimiento de las características del asentamiento, de forma de poder realizar la evaluación a través del instrumental teórico o sistema de variables y subvariables, aporte fundamental de esta investigación.
Para su confección se consideró la ponderación o peso de los indicadores y una escala de valores.

\section{Peso de indicadores o factor de ponderación}

El peso de los indicadores o factor de ponderación, permite expresar la importancia relativa de cada indicador componente del instrumental de evaluación, cuestión por la cual se hace necesario instrumentar en este proceso evaluativo.

Se otorgan tres categorías de importancia:

- Muy Importante: 3

- Importante: 2

- Menos importante: 1

\section{Escala de valores}

Aunque el método desarrollado es de carácter cualitativo, en él se utilizan procedimientos cuantitativos, por lo que se propuso una clasificación para determinar, cuantitativamente, el comportamiento de los componentes del medio construido y su influencia en la calidad ambiental. Para ello se utilizó la misma escala de valores empleada en las tablas, correspondientes a los parámetros evaluativos, desde 5 (excelente), hasta 2 (mal). A esta escala de valores se le denominó (E).

\section{Significado de las evaluaciones}

Se propusieron las diferentes situaciones evaluativas, que fueron ajustadas en cuanto al puntaje a partir del criterio de especialistas y actores, así como de los resultados de aplicación del instrumental, dándose rangos más cerrados en las condiciones de excelencia y más amplios en las peores condiciones a partir de estas consideraciones, del instrumental diseñado y validado por Gómez, (2005) y antes referido, se asume la siguiente escala de evaluación:

- Los valores de 5 en la escala, representan las situaciones más favorables o excelentes, estando los rangos evaluativos de 5 a 4,7 .

- Los valores de 4 en la escala representan situaciones con algunas afectaciones o limitaciones que afectan la calidad ambiental, estando los rangos evaluativos de 4,69 a 3,9.

- Los valores de 3 en la escala representan situaciones con afectaciones significativas en algunos de los componentes, que harán de tomar medidas que mitiguen o corrijan las situaciones que 


\section{F. del Pozo y Gómez G.}

degradan la calidad ambiental, estando los rangos evaluativos de 3,89 a 3 .

- Los valores de 2 en la escala, representan situaciones de elevada degradación de varios de los componentes de la calidad ambiental con medidas de consideración, estando los rangos evaluativos de 2,99 a 2 .

Al ser el método analizado de base cualitativa, aunque apoyado en análisis cuantitativos, como antes se expresó, se requiere de una valoración de los resultados cuantitativos que se obtengan en función del grado de importancia que puedan tener, al otorgar una evaluación de las que caigan en zonas extremas.

Con las evaluaciones teóricas de cada subvariable, se obtienen los valores cuantitativos para realizar la evaluación cualitativa, éstas son tomadas de las tablas de evaluación que contiene el instrumental y ponderadas a partir de los pesos de los indicadores adoptados y que fueron antes referidos, posibilitando la evaluación de cada variable, a partir del promedio expresado por la siguiente fórmula matemática:

\section{$\mathbf{E}=\Sigma \mathbf{E P} / \boldsymbol{\Sigma} \mathbf{P}$ Donde para cada subvariable: $\mathbf{E}$ es la evaluación \\ $\mathbf{P}$ es el peso o importancia y \\ EP la evaluación ponderada}

Se precisa, que al cambiar de asentamientos, pueden estos valores de ponderación tener alguna variación, que deberá ser ajustada por el equipo evaluador. A manera de ejemplo se presenta el diseño de un histograma correspondiente a una variable, para hacer objetiva la explicación desarrollada. (Ver gráfico No.1). Ejemplo de histograma.

Los resultados de los histogramas permiten conocer el comportamiento de cada variable, lo que posibilita determinar la problemática con especificidad, analizándose integralmente en la matriz síntesis.

\section{Desarrollo de la matriz}

Esta matriz sintetiza el valor de todos los factores, por lo que su diseño partió de la consideración de todas las variables, pudiendo llegarse a la evaluación resumen de los componentes del medio construido y su influencia en la calidad de vida para el asentamiento evaluado. Para la elaboración de la matriz, se utilizan colores que facilitan la visualización de los resultados:
- El verde intenso indica excelente.

- El verde claro de bien.

- El amarillo de regular.

- El rojo de mal.

Para la obtención del valor de cada variable que integra el instrumental de evaluación de la calidad ambiental en el asentamiento, se debe conocer el valor de las subvariables que las conforman y que fueron sintetizados en los histogramas, realizando simples procesos estadísticos (valor promedio).

Con el objetivo de determinar cuantitativamente el indicador de la evaluación, se partirá de la puntuación alcanzada, indicándose el cálculo a partir de la misma escala establecida en los histogramas. (Ver gráfico No.2). Ejemplo de matriz síntesis.

La matriz representa la síntesis de los resultados evaluativos, pudiendo visualizarse con claridad, en cuáles variables se presentan los más significativos problemas, lo que contribuye a la adopción de las medidas encaminadas a su solución o mitigación, en las instancias de competencia. Este paso se conforma a partir de un documento rector que a continuación se propone, el expediente de mejoramiento de la calidad ambiental para el planeamiento y la gestión. Con la realización de este expediente, se dará inicio al proceso de gestión, el mismo contemplará los diferentes pasos de un proceso de gestión:

- La identificación de los problemas ambientales determinados con el instrumental.

- La jerarquización de esos problemas.

- El plan de acciones y los mecanismos operacionales, encaminados a la adopción de lineamientos, realización de proyectos y programas, determinación de actores responsables, plazos de ejecución y formas de monitoreo, para dar respuesta a la problemática del medio construido, que afecta la calidad ambiental en los APP de la Provincia.

Se concede especial importancia a la gestión participativa, donde el rol de la comunidad asuma un papel principal. Las soluciones se gestarán en la base, incluyendo la capacitación de la comunidad en la comprensión del logro de la sustentabilidad, el autofinanciamiento y la mano de obra, siendo aspectos determinantes. 
Gráfico No.1. Ejemplo de histograma

\begin{tabular}{|c|c|c|c|c|c|c|c|}
\hline \multicolumn{8}{|c|}{ VARIABLE: 4 SERVICIOS SOCIALES } \\
\hline \multirow[t]{2}{*}{ SUBVARIABLES } & \multicolumn{5}{|c|}{$\begin{array}{c}\text { EVALUACIÓN } \\
\text { E }\end{array}$} & \multirow{2}{*}{$\begin{array}{c}\text { PESO O } \\
\text { IMPORTANCIA } \\
\text { P }\end{array}$} & \multirow{2}{*}{$\begin{array}{c}\text { EVALUACIÓN } \\
\text { PONDERADA } \\
\text { EP }\end{array}$} \\
\hline & 2 & 3 & 4 & 5 & & & \\
\hline Comercio y gastronomía & & & & & 3 & 3 & 9 \\
\hline Salud & & & & & 5 & $\mathbf{3}$ & 15 \\
\hline Educación & & & & & 4 & 3 & 12 \\
\hline Cultura, deporte y recreación & & & & & 2 & 2 & 4 \\
\hline SUMATORIAS & & & & & & $\Sigma \mathbf{P} 11$ & $\Sigma$ EP 38 \\
\hline VALOR TOTAL $E=\Sigma E P / \Sigma P$ & & & & & & & 3,45 Regular \\
\hline
\end{tabular}

Fuente: Histograma elaborado por Del Pozo, (2009), partiendo del de Gómez, (2005).

Gráfico No.2. Ejemplo de matriz síntesis

\begin{tabular}{|l|c|c|c|c|c|}
\hline \multirow{2}{*}{ VARIABLES } & \multicolumn{4}{|c|}{ EVALUACIÓN DE VARIABLES } \\
\hline & $\mathrm{M}$ & $\mathrm{R}$ & $\mathrm{B}$ & $\mathrm{E}$ & EVAL. \\
\hline FUNCIONALIDAD DEL ASENTAMIENTO & & & & & $\mathbf{2 , 6 0}$ \\
\hline ASENTAMIENTO Y SU ENTORNO NATURAL & & & & & $\mathbf{4 , 0 7}$ \\
\hline PERCEPCIÓN DEL ASENTAMIENTO & & & & & $\mathbf{3 , 5 1}$ \\
\hline SISTEMA DE SERVICIOS SOCIALES & & & & & $\mathbf{3 , 4 5}$ \\
\hline SISTEMA DE INFRAESTRUCTURAS TÉCNICAS & & & & $\mathbf{2 , 8 0}$ \\
\hline CARACTERÍSTICAS DE LAS VIVIENDAS & & & & $\mathbf{2 , 0 0}$ \\
\hline EVALUACIÓN DE LA CALIDAD AMBIENTAL & & $\mathbf{3 . 0 7}$ Regular \\
\hline
\end{tabular}

Fuente: Matriz elaborada por Del Pozo, (2009).

El instrumental presentado es de alta significación para evaluar la calidad ambiental en APP existentes, pudiendo utilizarse además en el planeamiento o diseño de este tipo de urbanización. Se recomienda que al ser implementado, sean consideradas las características particulares de cada territorio.

\section{CONCLUSIONES}

El instrumental diseñado para la evaluación de la calidad ambiental en los APP, está soportado en los conceptos de ambiente $y$ de sustentabilidad y constituye una herramienta de trabajo, para arquitectos y urbanistas, trabajadores sociales, líderes comunitarios, representantes de la dirección del estado, instituciones, y otros, que puedan accionar en el mejoramiento de la calidad ambiental, a través de la evaluación de los APP en funcionamiento y del planeamiento de los de nueva creación.

Se pretende que a partir de los resultados evaluativos con este instrumental, se posibiliten adoptar medidas en los referidos Asentamientos Poblacionales Periféricos (APP), para obtener una mayor calidad ambiental, es decir, medidas que atenúen el aumento de los desastres como consecuencia de causas naturales y antrópicas. Se remarca que la gestión del riesgo sólo puede alcanzarse mediante una nueva visión cultural, que incluya a todos los actores sociales, que posibilite que actúen frente a la problemática. Dando interés principal a la posterior 


\section{F. del Pozo y Gómez G.}

gestión participativa en la fase de implementación de las propuestas.

\section{REFERENCIAS}

Aguiló, Miguel; y otros: Guía para la elaboración de estudios del medio ambiente. MOPT, p. 808, Madrid, España, 1993.

"Asentamientos Sustentables en Cuba. Experiencias y Perspectivas", Arquitectura y Urbanismo, no 4, pp. 5560, ISPJAE, La Habana, Cuba, 1998.

Bancrofft, Rubén: "Desarrollo Sostenible Aplicado a la Construcción", Conferencia Magistral impartida en la 4ta Conferencia de la Vivienda y el Urbanismo, La Habana, Cuba.2002.

Castells, Manuel: La Cuestión Urbana. Siglo XXI Editores. México. 1988.

Conteras y Cordero Instrumental de evaluación de calidad de vida. Venezuela 1994.

Echarri, Víctor: Conocer nuestra arquitectura. Invariantes formales de la arquitectura tradicional de $\mathrm{La}$ Vega Baja del Segura, Diputación provincial de Alicante, España. 2001.

Dávila, Julio D: El estado del medio ambiente en las ciudades latinoamericanas, Estudios demográficos y urbanos, Vol. 13, No. 1, enero-abril, pp. 49-78, 1998.

Del Pozo, Fredy: "Instrumental para la evaluación ambiental en Asentamientos Poblacionales Periféricos (APP) de la ciudad de Santiago de Cuba", tesis en opción al título de Master en Ciencias Técnicas. Tutora Dra. Arq. Graciela Gómez. Universidad de Oriente. Cuba, 2010.

DPPF de Santiago de Cuba. Investigación realizada por el Grupo PGOU. Autores: Arq. Teresa Fernández, Arq. Yoanka Ramón. Arq. José Luís Peña e Ing. Teresa Massabeaut. 2008.

Environmental Building News, http://www.ebuild.com, 1999.

Girardet, H: Creando ciudades sostenibles. Valencia, 2001.

Gómez, Graciela: "Instrumental para la evaluación del medio construido en asentamientos rurales de Santiago de Cuba”, tesis en opción al grado científico de Doctor en Ciencias Técnicas. Tutor Dr. Arq. Rubén Bancrofft, CUJAE, Habana, Cuba, 2005.

González, Dania: "Medio ambiente construido y desarrollo sustentable", Folleto, Facultad de Arquitectura, ISPJAE, La Habana, Cuba, 2000.

Hernández, Heimdall: "Instrumental metodológico para la valoración de la calidad ambiental del medio construido en asentamientos rurales del municipio de Masaya, Nicaragua”, tesis en opción al grado científico de Doctor en Ciencias Técnicas. Tutora Dra. Arq. Graciela Gómez. Universidad Nacional de Ingeniería, Nicaragua, 2008.

Liepach, Katharina, Julia Sixt y Wolfgang Irrek: "Komunale Nachhalttigkeits-indikatoren" Wissenschaftszentrum, Nordrhein-Westfalen, Alemania, 2003.

PNUMA/ UICN/WWF: "Cuidar la tierra para el futuro de la vida", Departamento de medio ambiente, Universidad de Leicester Inglaterra, 1993.

"¿Que cambiara con la cumbre de Johannesburgo?, Ramírez Velarde, Luís. Introducción a la planificación territorial regional y urbana. Universidad de Cochabamba. Bolivia. 2002.

Notario, R y otros: "Economía de la construcción, editorial ISPJAE, La Habana, Cuba, 1987.

Zhang, Mingshun: Measuring Urban Sustainable in China, Institute of Environment Protection Bureau, Rotterdam, 2002.

Zúñiga, Alicia: "Instrumental metodológico para la valoración del medio construido y su influencia en la calidad de vida en asentamientos urbanos del municipio de Masaya, Nicaragua". Tutora Dra. Arq. Graciela Gómez. Universidad Nacional de Ingeniería, Nicaragua, 2008. 


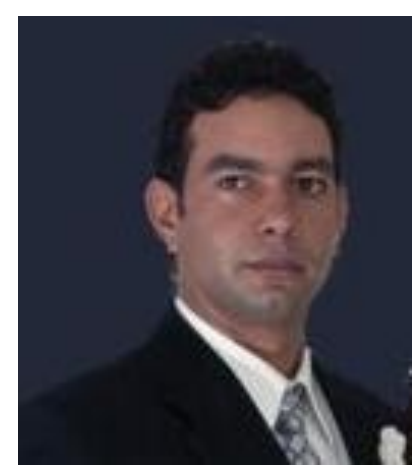

Fredy Del Pozo Gómez. Se graduó de Arquitecto en 2004, en la Universidad de Oriente. Cuba. Obtuvo el grado Académico de Master en Hábitat y Medio Ambiente en Zonas Sísmicas. Universidad de Oriente 2010. Es proyectista Arquitectónico y Urbano. Avenida de las Américas esquina a calle L. Su área de investigación es el urbanismo y el ambiente.

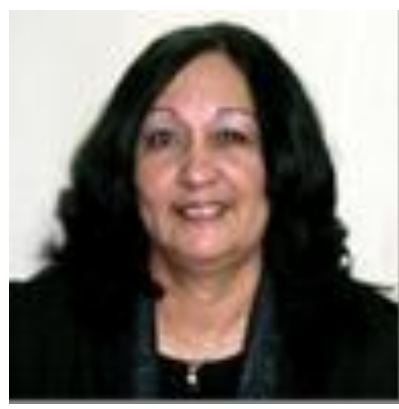

Graciela Gómez Ortega. Graduada de Arquitecta en la Universidad de Oriente. Cuba 1975. Obtuvo el grado Académico de Master en Medio Ambiente y Desarrollo Urbano en la Universidad Nacional de Ingeniería. Nicaragua 1999. Obtuvo el título científico de Dra. Ciencias Técnicas, en la CUJAE. Habana, Cuba. 2005. Es Profesora Titular Consultante. Miembro del comité académico de la Maestría en Hábitat y Medio Ambiente en Zonas Sísmicas. Universidad de Oriente. Cuba. Miembro del Comité Académico del doctorado en Ciencias del Ambiente del PEAUT de la Universidad Nacional de Ingeniería. Managua. Nicaragua. Miembro del Tribunal Permanente de Grados Científicos de Arquitectura y Urbanismo. Cuba. 